\title{
Endoscopic resection and ablation versus esophagectomy for high-grade dysplasia and intramucosal adenocarcinoma
}

\author{
Jörg Zehetner, MD, Steven R. DeMeester, MD, Jeffrey A. Hagen, MD, Shahin Ayazi, MD, \\ Florian Augustin, MD, John C. Lipham, MD, and Tom R. DeMeester, MD
}

\begin{abstract}
Background: Esophagectomy has been the traditional therapy for high-grade dysplasia and intramucosal adenocarcinoma. New endoscopic approaches allow treatment of these lesions with esophageal preservation. The aim of this study was to compare the outcome of endoscopic therapy with esophagectomy for high-grade dysplasia and intramucosal cancer.
\end{abstract}

Methods: A retrospective review was performed of all patients treated for high-grade dysplasia or intramucosal adenocarcinoma from 2001 to April 2010.

Results: Endoscopic therapy was performed in 40 patients (high-grade dysplasia $=22$, intramucosal cancer $=18$ ) and esophagectomy in 61 patients (high-grade dysplasia $=13$, intramucosal cancer $=48$ ). Endotherapy consisted of 102 endoscopic resections and 79 mucosal ablations (median 3 interventions per patient). In the endotherapy group, intramucosal cancer was completely resected in all patients. At last assessment, 10 patients have been converted to intestinal metaplasia without dysplasia and 21 to no residual intestinal metaplasia. Five patients have follow-up biopsy procedures pending after recent ablation, and esophagectomy was performed in 3 patients for failed endotherapy. A laparoscopic Nissen fundoplication has been performed in 8 patients after eradication of intestinal metaplasia. Esophagectomy resected the mucosal disease with negative margins in all patients. Compared with esophagectomy, endotherapy was associated with significantly lower morbidity $(39 \%$ vs $0 ; P<.0001)$ and similar survival $(94 \%$ at 3 years in both groups; median follow-up 34 months after esophagectomy vs 17 months after endotherapy; $P=.0026$ ).

Conclusions: Endoscopic therapy for high-grade dysplasia or intramucosal cancer has lower morbidity than an esophagectomy and similar survival during short-term follow-up, but required multiple procedures in most patients. Both therapies are appropriate options, but preservation of the esophagus allows the option of a fundoplication for reflux control, perhaps further improving long-term quality of life. (J Thorac Cardiovasc Surg 2011;141:39-47)

The incidence of esophageal adenocarcinoma and its precursor lesion, Barrett's esophagus, continues to increase in the United States and other Western countries. ${ }^{1-4}$ Surveillance programs have identified an increasing number of patients with high-grade dysplasia or intramucosal adenocarcinoma, for whom traditional therapy has been an esophagectomy. Although usually curative, esophagectomy is associated with considerable early and long-term morbidity.

Recently, the introduction of endoscopic techniques for resection of superficial lesions and ablation of the Barrett's mucosa has allowed the option of esophageal preservation in patients with high-grade dysplasia or intramucosal cancer.

\footnotetext{
From the Department of Surgery, Keck School of Medicine, University of Southern California, Los Angeles, Calif.

Disclosures: Authors have nothing to disclose with regard to commercial support.

Read at the 36th Annual Meeting of The Western Thoracic Surgical Association,

Ojai, California, June 23-26, 2010.

Received for publication June 8, 2010; revisions received July 20, 2010; accepted for publication Aug 14, 2010; available ahead of print Nov 9, 2010.

Address for reprints: Steven R. DeMeester, MD, Associate Professor of Cardiothoracic Surgery, University of Southern California, 1510 San Pablo St, Suite 514,

Los Angeles, CA 90033 (E-mail: sdemeester@surgery.usc.edu).

$0022-5223 / \$ 36.00$

Copyright (C) 2011 by The American Association for Thoracic Surgery

doi:10.1016/j.jtcvs.2010.08.058
}

The largest series of patients treated endoscopically for these lesions were reported from gastroenterology units in Wiesbaden and Amsterdam. ${ }^{5-7}$ Morbidity was low and survival excellent in these series; however, they are uncontrolled in that patients with the more complex conditions may have been referred for surgical therapy. We adopted endoscopic therapy in 2001, and the aim of this study was to compare the morbidity, mortality, survival, and cancer recurrence rates in concurrent patients with high-grade dysplasia or intramucosal cancer treated either with endoscopic therapy or esophagectomy in a surgical unit.

\section{PATIENTS AND METHODS}

We performed a retrospective review of the records of all patients with high-grade dysplasia or intramucosal adenocarcinoma treated endoscopically or by an esophagectomy at our institution from 2001 (when we started doing endoscopic therapy) through April 2010. Patients with tumors invasive into the submucosa were excluded, but lymphovascular invasion or poor differentiation in an intramucosal lesion did not deter endoscopic therapy. This study was approved by the Institutional Review Board of the University of Southern California.

\section{Evaluation and Definitions}

All patients were evaluated with upper endoscopy. Barrett's esophagus was defined as an endoscopically visible segment of columnar mucosa with 


\section{Abbreviations and Acronyms \\ $\mathrm{CT}=$ computed tomography \\ $\mathrm{IQR}=$ interquartile range \\ $\mathrm{PDT}=$ photodynamic therapy \\ $\mathrm{PET}=$ positron emission tomography}

intestinal metaplasia on histologic examination, and it was considered long segment if the length of columnar mucosa was $3 \mathrm{~cm}$ or longer. Biopsy specimens were taken in all patients from the antrum and gastric body, gastroesophageal junction, and every 1 to $2 \mathrm{~cm}$ from the gastroesophageal junction to the squamocolumnar junction. Additional biopsy samples were taken from any nodules or ulcers within the columnar mucosa. Patients with confirmed cancer were selectively evaluated with endoscopic ultrasound for locoregional staging and computed tomography (CT) and positron emission tomography (PET) scans for systemic staging. Patients considered for endoscopic therapy had a videoesophagogram to evaluate for the presence and size of a hiatal hernia and assess esophageal body bolus transport function when warranted by symptoms.

\section{Technique of Endoscopic Therapy}

All endoscopic resections were performed as an outpatient procedure in the operating room under general anesthesia by thoracic/foregut surgeons. Visible lesions were excised with endoscopic resection, and the depth of invasion was pathologically determined in the fixed specimen. Endoscopic resection was performed by the Inoue cap technique with either the straight $13.9-\mathrm{mm}$ or the soft, oblique 18-mm Olympus cap (Olympus Corporation, Shinjuku-ku, Japan) after dilute epinephrine in saline was injected into the submucosa to lift the lesion. ${ }^{8}$ Multiple resections were performed at a single setting, but never circumferential or exceeding $3 \mathrm{~cm}$ in height at one site.

Ablation was usually performed with either the Halo 360 or the Halo 90 (Halo systems; BARRX Medical Inc, Sunnyvale, Calif) radiofrequency catheters at the recommended energy setting $\left(12 \mathrm{~J} / \mathrm{cm}^{2}\right)$. Occasionally, argon plasma coagulation was used to touch up small areas, typically at the time of endoscopic resection of a lesion. In some patients immediately after endoscopic resection of a nodule, the surrounding Barrett's mucosa was ablated with the Halo 90 device. Ablation was not performed over areas just resected.

\section{Follow-up After Endoscopic Therapy}

An endoscopy with biopsies was done 8 weeks after endoscopic resection or ablation. Biopsy specimens were taken using the same protocol as before therapy, but in addition, samples of the neosquamous mucosa were taken, looking for buried glands, in areas where columnar mucosa had been. If residual Barrett's esophagus was present, most patients were immediately scheduled for further endoscopic resection or ablation. If no intestinal metaplasia was present, repeat endoscopy was done every 3 months for 1 year, then every 6 months for 1 year, and then annually. Elimination of intestinal metaplasia was accepted only after 2 consecutive endoscopies with biopsies showed no intestinal metaplasia and no buried glands. Patients with cancer were followed up clinically, and posttherapy endoscopic ultrasound, CT, and PET scans were not routinely obtained.

\section{Reflux Management}

During endoscopic therapy, patients were maintained on twice a day proton pump inhibitor therapy. Once all the intestinal metaplasia was eradicated, our preference was to perform a Nissen fundoplication for longterm reflux control and protection of the neosquamous mucosa. Before antireflux surgery, an esophageal manometry was performed as previously described. ${ }^{9}$

\section{Esophagectomy}

Esophagectomy was performed as a transthoracic en bloc, transhiatal, minimally invasive, or vagus-sparing resection as previously described. ${ }^{10,11}$ Reconstruction in all cases was with a tubularized gastric pull-up. Patients were followed up clinically by the operating surgeon after the esophagectomy for any evidence of recurrence, with selective use of CT or PET scans.

\section{Classification of Complications}

Complications were classified as either minor or major. Minor complications were defined as those not requiring interventional procedures or reoperations. Anastomotic leaks after esophagectomy were classified as major complications and divided into minor leaks if treated with antibiotics alone without interventional procedures, stenting, or reoperation. A leak requiring any intervention was classified as a major leak. Procedure-related mortality consisted of any death within 30 days or before hospital discharge.

\section{Statistical Analysis}

Data are expressed as medians and interquartile range (IQR). Comparisons of proportions were performed using the Fisher exact test. Continuous variables were compared using the Mann-Whitney test. Survival was calculated by the Kaplan-Meier method and compared using the log-rank method.

\section{RESULTS}

Endoscopic therapy was performed in 40 patients and esophagectomy in 61 patients (Table 1). The median age, gender, and length of Barrett's esophagus was similar in both groups. The prevalence of high-grade dysplasia was significantly higher in the endotherapy group, whereas intramucosal cancer was more prevalent in the esophagectomy group. There was no significant difference in the prevalence or types of comorbid conditions. Median follow-up was significantly longer after esophagectomy (endoscopic therapy: 17 months [IQR, 9-29 months]; esophagectomy: 34 months [IQR, 19-51 months]; $P=.0026$ ).

\section{Endoscopic Therapy}

Procedure. The 40 patients with endoscopic therapy had a median of 3 interventions per patient, consisting of 102 endoscopic resections and 79 mucosal ablations. The maximum number of treatment sessions was 8 in 1 patient. The endoscopic resections were performed in 53 sessions with 1 to 3 specimens retrieved for histopathologic analysis at each session. Mucosal ablation was performed with the $360^{\circ}$ radiofrequency balloon 16 times, with the $90^{\circ}$ device 51 times, and with the argon plasma coagulator 12 times.

Complications. There were no perforations during endoscopic resection or ablation, and no patient had bleeding requiring endoscopy or transfusion. All patients were discharged home on the day of the procedure. No patient had a stricture that required dilatation. Mild chest discomfort was common for several days after ablation, but there were no other procedure-related complications or symptoms. On follow-up endoscopic biopsies, buried Barrett's tissue was found in only 1 patient who had had ablation with argon plasma coagulation. No buried Barrett's tissue 
TABLE 1. Patient characteristics

\begin{tabular}{|c|c|c|c|}
\hline & $\begin{array}{c}\text { Endoscopic } \\
\text { therapy } \\
\text { group }(n=40)\end{array}$ & $\begin{array}{l}\text { Esophagectomy } \\
\text { group }(n=61)\end{array}$ & $\begin{array}{c}P \\
\text { value }\end{array}$ \\
\hline Median age in years (IQR) & $66(58-76)$ & $68(58-75)$ & .84 \\
\hline Gender male/female & $33 / 7$ & $49 / 12$ & 1.0 \\
\hline $\begin{array}{c}\text { Median length of Barrett's } \\
\text { esophagus, cm (IQR) }\end{array}$ & $3(2-5)$ & $4(2-9)$ & .16 \\
\hline $\begin{array}{l}\text { Prevalence of long-segment } \\
\text { Barrett's esophagus }\end{array}$ & $46 \%$ & $64 \%$ & .15 \\
\hline \multicolumn{4}{|l|}{ Histology before therapy } \\
\hline High-grade dysplasia & $22(55 \%)$ & $13(21 \%)$ & .0007 \\
\hline Intramucosal cancer & $18(45 \%)$ & $48(79 \%)$ & .0007 \\
\hline Lymphovascular invasion & $6 \%$ & $2 \%$ & .44 \\
\hline Poor differentiation & $6 \%$ & $8 \%$ & 1.0 \\
\hline Visible lesion & $22(55 \%)$ & $49(80 \%)$ & .0081 \\
\hline \multicolumn{4}{|l|}{ Comorbid conditions } \\
\hline Cardiac disease & $11(28 \%)$ & $22(36 \%)$ & .40 \\
\hline Respiratory disease & $17(43 \%)$ & $20(33 \%)$ & .40 \\
\hline Hypertension & $23(58 \%)$ & $32(52 \%)$ & .69 \\
\hline Renal disease & $3(8 \%)$ & $7(11 \%)$ & .74 \\
\hline Diabetes mellitus & $7(18 \%)$ & $7(11 \%)$ & .40 \\
\hline Tobacco abuse & $16(40 \%)$ & $27(44 \%)$ & .69 \\
\hline $\begin{array}{l}\text { Prevalence of any comorbid } \\
\text { condition }\end{array}$ & $80 \%$ & $82 \%$ & .80 \\
\hline
\end{tabular}

has been detected after endoscopic resection or radiofrequency ablation.

\section{Outcome}

Intramucosal cancer. Eighteen patients were treated endoscopically for an intramucosal cancer (Figure 1, $A$ ). In 16 patients a visible lesion was present, and all had endoscopic resection of the lesion. All resection margins were negative. In 11 patients invasion was into the lamina propria whereas in 5 there was invasion into but not through the muscularis mucosa. After initial endoscopic resection, 1 patient on rebiopsy had no residual Barrett's tissue whereas the other 15 patients underwent additional endoscopic procedures for residual Barrett's esophagus with or without dysplasia. One of these 15 patients ultimately had an esophagectomy for persistent high-grade dysplasia after 3 endoscopic procedures. The final pathologic report on the resected specimen in this patient showed multifocal highgrade dysplasia without adenocarcinoma.

There was no visible lesion in 2 patients with a biopsy showing adenocarcinoma, and both patients were treated with radiofrequency ablation. Subsequent endoscopy in 1 of the patients showed a lesion that was endoscopically resected and proved to be an intramucosal cancer. Biopsy specimens in this patient after further endotherapy now show no intestinal metaplasia. The other patient is still undergoing ablation for residual intestinal metaplasia, but there is no dysplasia.

None of the 17 patients with intramucosal cancer treated endoscopically without esophagectomy progressed to submucosal cancer, but metachronous cancers developed in $3(18 \%)$ patients. All 3 patients had residual Barrett's esophagus or dysplasia on biopsy specimens immediately before the identification of the cancer. At the time of the last follow-up endoscopic examination, $9(53 \%)$ of the 17 patients no longer had intestinal metaplasia and $14(82 \%)$ were free of dysplasia or cancer. A laparoscopic Nissen fundoplication had been performed in 2 patients treated endoscopically for intramucosal cancer to correct the pathophysiology of their reflux disease.

High-grade dysplasia. Twenty-two patients were treated endoscopically for high-grade dysplasia (Figure 1, B). Endoscopic resection was performed in 10 patients, 6 with a visible lesion that proved not to be cancer after resection and 4 with a short tongue of Barrett's esophagus believed to be amenable to complete endoscopic resection. The other 12 patients underwent radiofrequency ablation as the initial therapy. Additional endoscopic procedures were necessary in 13 patients. Of these 22 patients, 1 died of causes unrelated to esophageal disease, and 2 had an esophagectomy for persistent high-grade dysplasia. Among the remaining 19 patients, progression to cancer occurred in $5(26 \%)$ before complete ablation of intestinal metaplasia, and all were successfully treated with endoscopic resection. In 4 patients the cancer was intramucosal and in 1 it was submucosal. All endoscopic resection margins were clear, no patient has had recurrent cancer, and no patient went on to esophagectomy (the patient with submucosal invasion declined esophagectomy and at 12 months of follow-up remains free of disease). At the time of last biopsy, 17 (89\%) of the 19 patients treated endoscopically for high-grade dysplasia had either no intestinal metaplasia $(n=12)$ or intestinal metaplasia without dysplasia $(n=5)$. From this group, 6 patients have had a Nissen fundoplication.

Length of Barrett's esophagus and success with endoscopic therapy. The median length of Barrett's esophagus before endoscopic therapy was $3 \mathrm{~cm}$ (range, 1-11 cm). In the 21 patients with complete eradication of intestinal metaplasia, the median number of treatment sessions necessary was significantly fewer in patients with short- versus longsegment Barrett's esophagus (median 2 for short segment vs 5 for long segment; $P=.047$ ). The median time to complete eradication of intestinal metaplasia was 20 months (IQR, 14-28 months). The relationship between length of Barrett's esophagus and the number of treatment sessions is shown in Figure 2.

\section{Primary Esophagectomy}

Procedure. Esophagectomy was done for high-grade dysplasia in 13 patients and intramucosal adenocarcinoma in 48 patients. The esophagectomy was done as a transthoracic en bloc procedure in 11 patients, a transhiatal procedure in 20 patients, and a vagus-sparing procedure in 30 patients. In 10 patients, the vagus-sparing esophagectomy was 


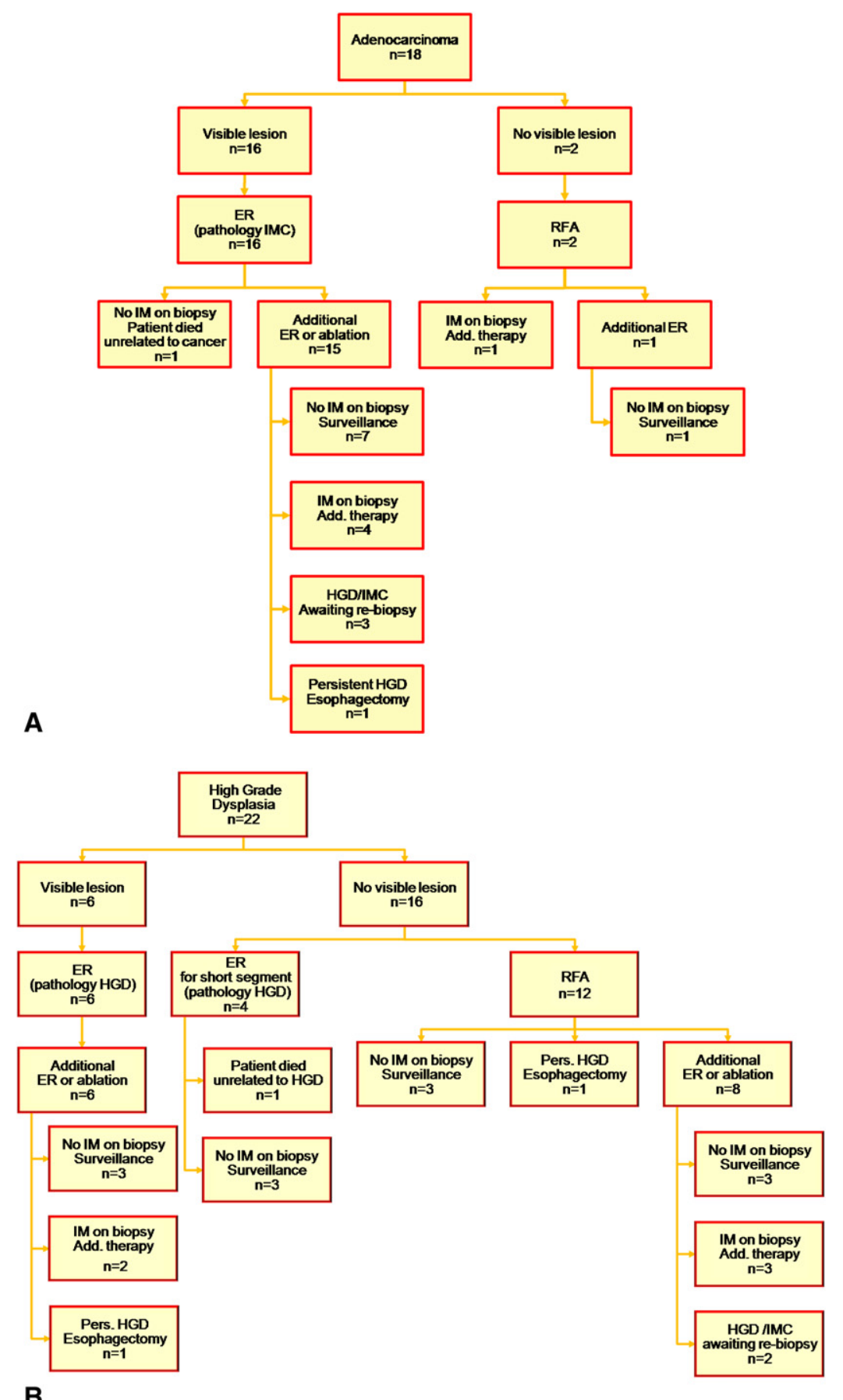

FIGURE 1. A, Flow chart for the 18 patients with intramucosal cancer. B, Flow chart for the 22 patients with high-grade dysplasia. ER, Endoscopic resection; RFA, radiofrequency ablation; $I M$, intestinal metaplasia; $H G D$, high-grade dysplasia; $I M C$, intramucosal cancer.

done laparoscopically. During the years of the study, the frequency of an en bloc resection decreased whereas the vagus-sparing esophagectomy increased. A staging endoscopic resection had been performed in $20(33 \%)$ patients before vagus-sparing esophagectomy to confirm the depth of invasion of a visible lesion. In the 48 patients with intramucosal cancer, a total of 921 lymph nodes were removed, and only 1 node in 1 patient was positive for metastatic cancer by routine histologic examination $(2.1 \%)$.

Complications. Postoperative complications occurred in $39 \%$ of patients after esophagectomy (Table 2). There was no mortality, including the 3 patients that had esophagectomy for failed endoscopic therapy. Long-term complications occurred in $61 \%$ of patients and included 


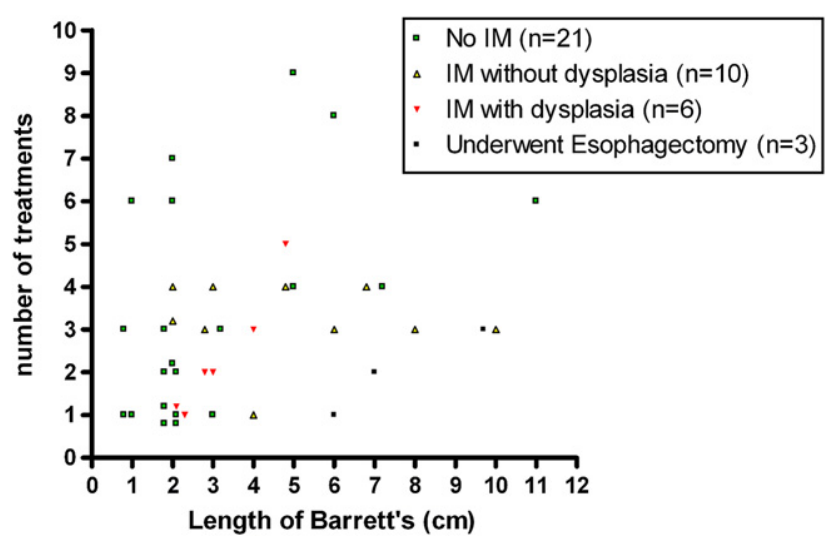

FIGURE 2. Length of Barrett's segment before endoscopic therapy, number of treatment sessions, and pathology at the time of most recent endoscopy and biopsy. $I M$, intestinal metaplasia.

anastomotic strictures, ventral hernias, reflux, and postprandial dumping or diarrhea.

Outcome. After esophagectomy, all resection margins were free of intestinal metaplasia. In $1(8 \%)$ patient who had esophagectomy for high-grade dysplasia, a focus of intramucosal cancer was found in the resected specimen, and among the 48 patients who had an esophagectomy for confirmed adenocarcinoma, multifocal cancer was found in the resected specimen in $1(2 \%)$ patient. One patient with intramucosal cancer but no lymph node metastases died of systemic disease at 50 months after esophagectomy. No other cancer recurrences have been detected, including the only patient who had an involved lymph node at the time of resection.

\section{Comparison of Endotherapy With Esophagectomy}

Over the years of the study there has been a shift at our center from esophagectomy to endoscopic therapy for

TABLE 2. Complications after esophagectomy for high-grade dysplasia or intramucosal adenocarcinoma $(n=61)$

\begin{tabular}{lc}
\hline Perioperative complications & \\
Minor & $16(26 \%)$ \\
Atrial fibrillation & 6 \\
Wound infections & 7 \\
Others & 3 \\
Major & $8(13 \%)$ \\
$\quad$ Leaks: minor/major & $2 / 0(3.3 \%)$ \\
$\quad$ Wound dehiscence & 2 \\
Others & 4 \\
Total & $24(39 \%)$ \\
Long-term complications & \\
Dumping & $13(21 \%)$ \\
Diarrhea & $14(23 \%)$ \\
Stricture requiring dilatation & $17(28 \%)$ \\
Reflux symptoms & $36(59 \%)$ \\
Nocturnal aspiration events & $17(28 \%)$ \\
Reoperation for incisional hernia & $7(11 \%)$ \\
Total & $37(61 \%)$ \\
\hline
\end{tabular}

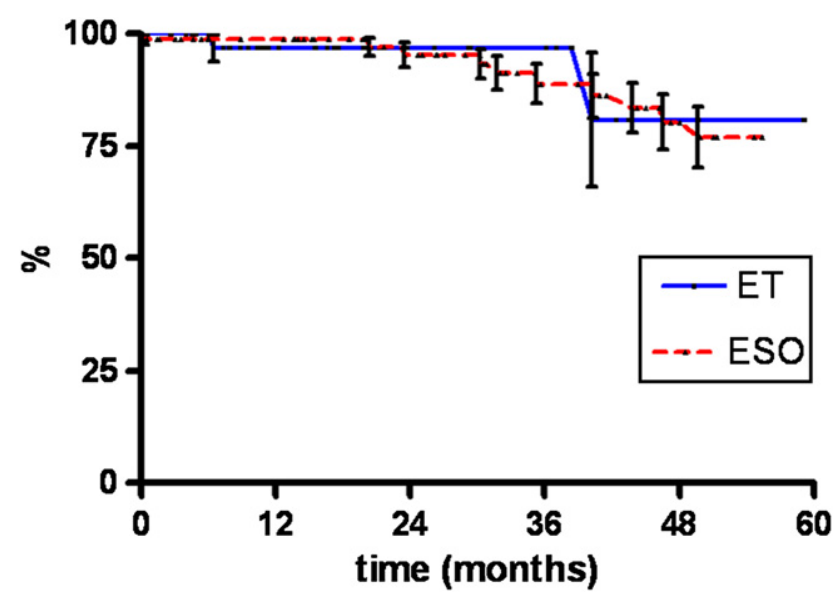

FIGURE 3. Kaplan-Meier curve of overall survival for endoscopic therapy $(E T)$ and esophagectomy $(E S O)$.

high-grade dysplasia and intramucosal cancer, and currently approximately $80 \%$ of patients are treated endoscopically. Early morbidity was significantly higher after esophagectomy ( $39 \%$ vs $0 ; P<.0001)$; however, there was no procedure-related mortality with either therapy. A new or metachronous cancer developed in $8(20 \%)$ patients undergoing endoscopic therapy but in no patient after esophagectomy. There was no difference in overall survival between endotherapy and esophagectomy ( $94 \%$ at 3 years in both groups; Figure 3). Cancer-related survival was $100 \%$ in both groups at 3 years and $100 \%$ in the endotherapy group compared with $88 \%$ in the esophagectomy group at 5 years $(P=.54)$.

Esophagectomy removed all the mucosal disease, and there has been no recurrence of Barrett's tissue in the residual cervical esophagus in any patient after esophagectomy. In the endotherapy group all intestinal metaplasia has been eradicated in $58 \%$ of patients, but the likelihood of complete eradication increased with the duration of therapy and follow-up. There were 26 patients who had endotherapy and follow-up for a minimum of 1 year. In this group, all intestinal metaplasia has been eradicated in $18(69 \%)$ patients. Among 10 patients with at least 3 years of followup, $80 \%$ have no intestinal metaplasia, and in the 4 patients with at least 5 years of follow-up, $100 \%$ have no intestinal metaplasia. Importantly, there has been no recurrence of dysplasia or cancer in patients from whom all intestinal metaplasia was eradicated, and none of these patients had intestinal metaplasia redevelop during a median follow-up of 8 months. A Nissen fundoplication has been performed in $8(20 \%)$ patients thus far after eradication of the mucosal disease with endoscopic therapy.

\section{DISCUSSION}

Esophageal adenocarcinoma remains a dreaded disease, known for poor survival and difficult and life-altering 
therapy. However, the increasing frequency of finding highgrade dysplasia or adenocarcinoma while still intramucosal and the introduction of effective endoscopic therapies for these lesions represent a major advance for the care and outcome in these patients. Initial attempts at endoscopic therapy in the 1980s were largely with photodynamic therapy (PDT) ${ }^{12-15}$ Problems with PDT included the lack of a specimen to confirm the depth of invasion if there was a visible lesion, the high rate of stricture and buried Barrett's tissue, and the phototoxicity that accompanied the procedure. The development of endoscopic resection techniques that allow visible lesions to be excised and the depth of invasion determined, as well as the introduction of radiofrequency ablation that avoids many of the problems with PDT, has launched endoscopic therapy into the forefront for the management of patients with highgrade dysplasia or superficial esophageal adenocarcinoma.

Before the extension of endoscopic therapy to the treatment of superficial esophageal adenocarcinoma, it was necessary for the biology of this tumor to be clearly defined. In particular, an understanding of the important relationship between the depth of tumor invasion and the risk of lymphatic metastases was critical. This information has been provided by a number of surgical series in which it was shown that tumors with invasion confined to the mucosa rarely $(<2 \%)$ have nodal metastases. ${ }^{11,16-18}$ Further, in a comparison of resection techniques, we ${ }^{11}$ showed that the type of esophagectomy and extent of lymphadenectomy did not affect survival for an intramucosal adenocarcinoma. Consequently, endoscopic resection is appropriate from an oncologic standpoint for tumors limited to the mucosa. In contrast, submucosal invasion is associated with an approximately $20 \%$ risk of nodal metastases, and therapies that do not address potentially involved lymph nodes are not appropriate. $^{19}$

Esophagectomy has been the traditional therapy for highgrade dysplasia and intramucosal cancer, and it is curative in nearly all patients. Further, operative mortality rates for these conditions, $0 \%$ to $1 \%$ in major centers, are much lower than in patients with locoregionally advanced disease. ${ }^{20}$ However, morbidity remains significant. Since lymphadenectomy is not necessary for patients with high-grade dysplasia or intramucosal cancer, we have scaled down the resection and now preferentially use a vagus-sparing procedure. This operation has reduced morbidity compared with a standard esophagectomy with vagal disruption and can be done laparoscopically. ${ }^{11}$ An advantage of esophagectomy, as shown in this series, is that the therapy is immediate and lasting. All resection margins were free of Barrett's tissue and no patient had intestinal metaplasia redevelop during follow-up nor required further intervention for their original condition. This is in stark contrast to the frequent follow-up endoscopies and repetitive interventions usually necessary in the endotherapy group. Further, although 1 pa- tient with a preoperative diagnosis of high-grade dysplasia was found to have a focus of invasive adenocarcinoma after esophagectomy, the other 12 patients had only high-grade dysplasia and are therefore at no risk of death from metastatic esophageal adenocarcinoma. In contrast, in the endotherapy group, $26 \%$ of patients who started therapy with only high-grade dysplasia progressed to invasive cancer and have incurred the potential risk, although very small, for death from metastatic disease.

Despite the immediacy and finality of treatment with esophagectomy, the potential for early and long-term complications as well as the magnitude of the procedure discourage patients from having the operation. This is reflected by the shift in management of patients with highgrade dysplasia and intramucosal adenocarcinoma seen at our center and other major esophageal centers worldwide, where most patients now undergo endoscopic therapy with the goal of preserving the esophagus. In our series, endotherapy was associated with a significant reduction in morbidity with equivalent short-term survival compared with an esophagectomy. In fact, the only death from cancer was in a patient who had an esophagectomy. We recognize that our study is limited by small numbers and shorter follow-up in the endotherapy group. Nonetheless, we have 4 patients who were endoscopically treated for an intramucosal cancer with follow-up to 3 years and 3 with follow-up to 5 years, and none has demonstrated metastatic disease. Further, large experiences from Amsterdam and Wiesbaden have shown excellent survival with endoscopic resection and ablation for high-grade dysplasia and intramucosal cancer. $^{5-7}$ The absence of cancer deaths in these series is surprising and suggests that perhaps a limited endoscopic resection has less impact on the immune system than an esophagectomy and further reduces the already small risk of metastatic disease in these patients. Alternatively, the follow-up in these series may be too short for micrometastatic disease in lymph nodes or elsewhere to become clinically evident. At this time, though, it is reasonable to conclude that the risk of metastatic disease is not higher with endoscopic therapy compared with an esophagectomy for intramucosal cancer.

Despite the willingness of most patients to have endoscopic therapy, in our opinion selection is important inasmuch as not every patient is an appropriate candidate for endoscopic therapy. There are 3 factors that we consider in our evaluation: tumor factors, esophageal factors, and patient factors (Table 3). We recommended esophagectomy to several patients in this series with multifocal high-grade dysplasia or intramucosal cancer thought to be too extensive for endotherapy, or with end-stage reflux disease in which esophageal preservation did not make sense. We also performed an esophagectomy in 3 patients after failed attempts at endotherapy. A vagus-sparing esophagectomy remains an excellent option for these patients and was not precluded by 
TABLE 3. Factors considered in the selection of patients for esophageal preservation with endotherapy

\begin{tabular}{l} 
Tumor factors \\
1. High-grade dysplasia or intramucosal cancer (no submucosal tumors \\
owing to risk of lymph node metastases) \\
2. Visible lesions resected with negative deep margins (positive lateral \\
margins acceptable with additional endotherapy) \\
3. All intestinal metaplasia can be successfully eradicated \\
Esophageal factors \\
1. Esophagus is worth saving (no end-stage reflux disease) \\
2. Reflux disease controlled with proton pump inhibitor medications (no \\
severe heartburn, regurgitation, aspiration, or dysphagia symptoms) \\
3. Acceptable bolus transport on videoesophagogram or peristalsis on \\
motility study (candidate for Nissen fundoplication) \\
Patient factors \\
1. Patient able to understand pros and cons of each approach \\
2. Patient able to live with the uncertainty of complete mucosal disease \\
eradication, potential for buried glands, and potential for cancer \\
development or recurrence, perhaps at more advanced stage \\
3. Patient willing and able to return to our center for repeat follow-up \\
endoscopies with or without additional endoscopic resections or \\
ablations on a frequent basis, at least early in the therapy \\
4. Patient understands that endotherapy may fail and they might require \\
esophagectomy anyway \\
5. Patient understands that antireflux surgery is recommended once all \\
intestinal metaplasia has been successfully eradicated \\
\hline
\end{tabular}

prior endoscopic resection or ablation. We had a policy of esophagectomy for persistent disease after 3 attempts at endotherapy, but in some cases when the initial radiofrequency ablation had minimal impact on the diseased mucosa we recommended an esophagectomy earlier. All of the patients who went on to esophagectomy had longsegment Barrett's esophagus $(6,7$, and $10 \mathrm{~cm})$, and long segments of Barrett's tissue are more difficult to eradicate than short segments. However, there were 7 patients with similar or longer segments of Barrett's tissue that were successfully ablated to no intestinal metaplasia or to nondysplastic Barrett's tissue, and consequently patients with long-segment Barrett's esophagus should not be excluded from an attempt at endotherapy. Another consideration is that radiofrequency ablation was the primary mode of ablation attempted in the 3 patients who went on to esophagectomy for failed endotherapy. It is possible that greater use of endoscopic resection might have allowed salvage of the esophagus in these patients. We avoided overlapping resections using the Inoue cap technique, and because of the concern for a "cookie-cutter" issue with nonoverlapping resections, where residual Barrett's tissue would be left between resection sites, we reserved endoscopic resection largely for removal of lesions. Recently, we have switched to the band ligator device for endoscopic resection, and this seems to permit safer and easier overlapping resections. Patients with areas of Barrett's esophagus refractory to radiofrequency ablation can have stepwise radical endoscopic resection, as others have reported..$^{21}$ Using this technique, we likely will be able to reduce the number of patients with failed endotherapy in the future. In addition, new ablation modalities such as cryoablation may treat areas refractory to radiofrequency energy. ${ }^{22}$

An advantage of endotherapy, in addition to the safety and low morbidity of the procedure, is the fact that ultimately reflux can be eradicated with an antireflux procedure, whereas after an esophagectomy patients have life-long potential for reflux, regurgitation, and aspiration. During endotherapy, we prefer intensive medical treatment for reflux and delay a fundoplication until all the intestinal metaplasia has been eradicated for approximately 1 year to avoid having to do an esophagectomy for recurrent or persistent disease after performing a fundoplication. This approach is perhaps too conservative, and early and effective control of reflux during endotherapy with a fundoplication may be beneficial, particularly in patients with refractory Barrett's esophagus or dysplasia. Future studies will provide insight into the optimal timing for antireflux surgery in these patients.

Although endotherapy is preferred by most patients, it is burdensome for both the patient and physician. The median number of procedures in our series was 3 , and in 1 patient 8 procedures were performed. The goal of endotherapy must be complete eradication of all intestinal metaplasia inasmuch as any residual intestinal metaplasia is at risk for progression. A new or metachronous intramucosal cancer developed in 8 of our patients while undergoing endotherapy before successful eradication of all intestinal metaplasia. In retrospect, in some of these patients the endotherapy schedule should have been more aggressive, because delays in scheduling another ablation accounted for some of the progression. This risk of metachronous tumor development was also seen in the series from Wiesbaden, where initially tumors were excised endoscopically but the residual Barrett's esophagus was not being ablated. ${ }^{6}$ Subsequently, ablation for the residual Barrett's esophagus has been added to their protocol, and cancer recurrence has been reduced. ${ }^{23}$ Given this risk, we recommend repeated resections and ablations on a 2-month schedule until all intestinal metaplasia has been eradicated. In patients in whom all the intestinal metaplasia or dysplasia cannot be eradicated, consideration should be given to an esophagectomy.

Despite the workload for endotherapy, it is associated with minimal complications and is well tolerated by patients. We have had no episodes of significant bleeding, no perforations, and no strictures. However, we limit the extent of resection at any one time and prefer to do repeat procedures rather than be overly aggressive at one setting. This may account for the safety and minimal complications we have observed. As the number of patients in various stages of treatment and follow-up increase, it becomes a significant burden on the office staff to keep them all organized, and the patients need to stay engaged in the process to make sure 
that their therapy stays on track. These issues are likely to be further magnified by an increasing number of patients undergoing endoscopic therapy and continued economic challenges in medicine, particularly related to the poor reimbursement for these procedures.

In conclusion, both endoscopic therapy and esophagectomy are appropriate treatment options for patients with high-grade dysplasia or intramucosal adenocarcinoma. Although morbidity is increased with esophagectomy compared with endotherapy, esophagectomy is generally a 1-shot treatment. In contrast, the majority of patients undergoing endoscopic therapy require multiple interventions and remain at risk for cancer development until all the intestinal metaplasia has been eradicated. Failure to aggressively eradicate all Barrett's tissue and carefully monitor these patients sets the stage for recurrent cancer, and could potentially change a largely curable condition into a fatal disease. Although endoscopic therapy has revolutionized the therapy for high-grade dysplasia and intramucosal cancer, it is critical to not forget the lethality of this cancer and get nonchalant in patients fortunate enough to be found with a lesion that is nearly always curable.

\section{References}

1. Pohl H, Welch HG. The role of overdiagnosis and reclassification in the marked increase of esophageal adenocarcinoma incidence. J Natl Cancer Inst. 2005;97: 142-6.

2. Alcedo J, Ferrandez A, Arenas J, Sopena F, Ortego J, Sainz R, et al. Trends in Barrett's esophagus diagnosis in Southern Europe: implications for surveillance. Dis Esoph. 2009;22:239-48.

3. Conio M, Cameron AJ, Romero Y, Branch CD, Schleck CD, Burgart LJ, et al. Secular trends in the epidemiology and outcome of Barrett's oesophagus in Olmsted County, Minnesota. Gut. 2001;48:304-9.

4. Rex DK, Cummings OW, Shaw M, Cumings MD, Wong RK, Vasudeva RS, et al. Screening for Barrett's esophagus in colonoscopy patients with and without heartburn. Gastroenterology. 2003;125:1670-7.

5. Ell C, May A, Gossner L, Pech O, Gunter E, Mayer G, et al. Endoscopic mucosal resection of early cancer and high-grade dysplasia in Barrett's esophagus. Gastroenterology. 2000;118:670-7.

6. Ell C, May A, Pech O, Gossner L, Guenter E, Behrens A, et al. Curative endoscopic resection of early esophageal adenocarcinomas (Barrett's cancer). Gastrointest Endosc. 2007;65:3-10.

7. Pouw RE, Gondrie JJ, Sondermeijer CM, ten Kate FJ, van Gulik TM, Krishnadath KK, et al. Eradication of Barrett esophagus with early neoplasia by radiofrequency ablation, with or without endoscopic resection. J Gastrointest Surg. 2008;12:1627-36; discussion 1636-7.

8. Inoue H, Takeshita K, Hori H, Muraoka Y, Yoneshima H, Endo M. Endoscopic mucosal resection with a cap-fitted panendoscope for esophagus, stomach, and colon mucosal lesions. Gastrointest Endosc. 1993;39:58-62.

9. Zaninotto G, DeMeester TR, Schwizer W, Johansson KE, Cheng SC. The lower esophageal sphincter in health and disease. Am J Surg. 1988;155:104-11.

10. Hagen JA, DeMeester SR, Peters JH, Chandrasoma P, DeMeester TR. Curative resection for esophageal adenocarcinoma: analysis of 100 en bloc esophagectomies. Ann Surg. 2001;234:520-30; discussion 530-1.

11. Peyre CG, DeMeester SR, Rizzetto C, Bansal N, Tang AL, Ayazi S, et al. Vagalsparing esophagectomy: the ideal operation for intramucosal adenocarcinoma and Barrett with high-grade dysplasia. Ann Surg. 2007;246:665-71; discussion 671-4.

12. Gossner L, Stolte M, Sroka R, Rick K, May A, Hahn EG, et al. Photodynamic ablation of high-grade dysplasia and early cancer in Barrett's esophagus by means of 5-aminolevulinic acid. Gastroenterology. 1998;114:448-55.

13. Overholt BF, Panjehpour M, Halberg DL. Photodynamic therapy for Barrett's esophagus with dysplasia and/or early stage carcinoma: long-term results. Gastrointest Endosc. 2003;58:183-8.
14. Overholt BF, Panjehpour M, Haydek JM. Photodynamic therapy for Barrett's esophagus: follow-up in 100 patients. Gastrointest Endosc. 1999;49:1-7.

15. Wani S, Puli SR, Shaheen NJ, Westhoff B, Slehria S, Bansal A, et al. Esophageal adenocarcinoma in Barrett's esophagus after endoscopic ablative therapy: a meta-analysis and systematic review. Am J Gastroenterol. 2009;104:502-13.

16. Oh DS, Hagen JA, Chandrasoma PT, Dunst CM, DeMeester SR, Alavi M, et al. Clinical biology and surgical therapy of intramucosal adenocarcinoma of the esophagus. J Am Coll Surg. 2006;203:152-61.

17. Rice TW, Blackstone EH, Goldblum JR, DeCamp MM, Murthy SC, Falk GW, et al. Superficial adenocarcinoma of the esophagus. J Thorac Cardiovasc Surg. 2001; 122:1077-90.

18. Stein HJ, Feith M, Bruecher BL, Naehrig J, Sarbia M, Siewert JR. Early esophageal cancer: pattern of lymphatic spread and prognostic factors for long-term survival after surgical resection. Ann Surg. 2005;242:566-73; discussion 573-5.

19. Leers JM, DeMeester SR, Oezcelik A, Klipfel N, Ayazi S, Abate E, et al. The prevalence of lymph node metastases in patients with T1 esophageal adenocarcinoma: a retrospective review of esophagectomy specimens. Ann Surg. 2010, (in press).

20. Ferguson MK, Naunheim KS. Resection for Barrett's mucosa with high-grade dysplasia: implications for prophylactic photodynamic therapy. J Thorac Cardiovasc Surg. 1997;114:824-9.

21. Pouw RE, Peters FP, Sempoux C, Piessevaux H, Deprez PH. Stepwise radical endoscopic resection for Barrett's esophagus with early neoplasia: report on a Brussels' cohort. Endoscopy. 2008;40:892-8.

22. Dumot JA, Vargo JJ II, Falk GW, Frey L, Lopez R, Rice TW. An open-label, prospective trial of cryospray ablation for Barrett's esophagus high-grade dysplasia and early esophageal cancer in high-risk patients. Gastrointest Endosc. 2009;70: 635-44.

23. Pech O, Behrens A, May A, Nachbar L, Gossner L, Rabenstein T, et al. Longterm results and risk factor analysis for recurrence after curative endoscopic therapy in 349 patients with high-grade intraepithelial neoplasia and mucosal adenocarcinoma in Barrett's oesophagus. Gut. 2008;57:1200-6.

\section{Discussion}

Dr Ross M. Bremner (Phoenix, Ariz). One year ago at this meeting we discussed the optimal treatment of patients with very early esophageal cancer at a breakfast meeting, referring of course to patients with high-grade dysplasia and intramucosal carcinoma. Since these patients rarely, if ever, have the potential for lymph node or metastatic spread, local therapies are probably appropriate. Our experiences with the techniques of endoscopic resection and radiofrequency and cryoablation are relatively limited. The work that we have heard this morning probably represents the largest US experience, and, although this is a retrospective review, I congratulate the authors on their impressive data and a very thoughtful and well-written manuscript. I do have a couple of questions.

One of the problems with embarking on an endoscopic protocol is relying on the biopsy protocols that we do inasmuch as they introduce a possible sampling error from the biopsy specimens. How do we know that we have sampled adequately and can we be reassured that we are not missing a more advanced cancer? In that regard, is there any value for narrow-band imaging, vital dyes, and perhaps high-frequency ultrasound to help our biopsy techniques?

Dr DeMeester. Yes, that is an important issue. I think narrowband imaging is probably useful. It is much easier than vital sprays and dyes, which are fairly complicated, time consuming, and a bit of a nuisance. The reality is, if you follow a Seattle protocol-4-quadrant biopsies every 1 to $2 \mathrm{~cm}$ throughout the length of the columnar mucosa-you do not miss many lesions. You get at least a feel for what is going on. I think it is less of an issue of missing high-grade dysplasia than actually the pathologist being wrong, and it is important to have a good pathologist interpret the pathology. 
In terms of when there is high-grade dysplasia, is there also an invasive cancer that you do not know about? Multiple surgical series of esophagectomies have shown that almost $50 \%$ of the time there will be an associated adenocarcinoma that has not been identified. However, a number of years ago John Nigro presented our paper on that. If you have a high-grade dysplasia and a focus of invasive cancer, in almost all cases the lesion is limited to the mucosa. It would be rare to have an invisible lesion penetrating into the submucosa, so you do not need to worry about that in general. You can reliably ablate high-grade dysplasia and nonvisible cancers with the techniques available today.

Dr Bremner. I agree that embarking on an endoscopic protocol is pretty labor intensive inasmuch as it requires multiple therapies and continued follow-up. Quite frankly, it is a little nerve-wracking when you start doing this on otherwise healthy younger patients. However, once cured, swallowing with one's own esophagus is intuitively better than swallowing with a gastric tube. Have you done any long-term quality of life indices to help us understand this better, or are you planning to do this once you have longer follow-up?

Dr DeMeester. That is an important issue, and I think we will pursue that down the road. However, anecdotally there is no comparison, particularly in the case of a patient who can then undergo a Nissen fundoplication. Regardless of how good the results are with the gastric pull-up or an esophageal replacement, these patients have lifestyle modifications and reflux disease the rest of their lives. They sleep with the head of their bed elevated, they have to eat their evening meal early, because they are at constant risk of regurgitation and aspiration, and that cannot be avoided. I think it is going to end up very favorable for esophageal preservation, but it is a big issue in terms of the number of treatment sessions necessary to get these patients to the point of having no intestinal metaplasia.

Dr Bremner. That is quite relevant, especially with the younger patients. There is going to be a paper discussed at this meeting regarding the incidence of Barrett's esophagus in patients who have had an esophagectomy in the proximal part of the esophagus. Most of these patients with high-grade dysplasia are going to be cured with an esophagectomy, but 10 to 20 years down the line, they may still have a problem with Barrett's esophagus.

Dr Robert Cerfolio (Birmingham, Ala). The follow-up of these patients is the key thing to me. When you have a patient who has a very low operative risk and you know you can probably get him through it and cure him, deciding to do something that is "more conservative" I would argue is less conservative. What followup schedule do you use? What is the frequency, and do you use endoscopic ultrasound routinely on your follow-up?

Dr DeMeester. No. Are you talking about an endoscopic patient?

Dr Cerfolio. Yes.

Dr DeMeester. We do not use endoscopic ultrasound at all because it is worthless for these early lesions. The tumors have to get deep into the submucosa or into the muscularis propria before they can be reliably identified even with high-frequency ultrasound, so I follow them up with routine endoscopies. They get an endoscopy at 8 weeks after their ablative procedure and biopsies. If there is any residual intestinal metaplasia or dysplasia, they are immediately scheduled for another ablative therapy, and that sequence continues every 8 weeks until we get rid of all the intestinal metaplasia.

We have learned the hard way. A few patients have had delays in their follow-up and they have come back then with progression to high-grade dysplasia or cancer.

Dr Cerfolio. That has been our problem as well and hence the question. The compliance with follow-up is another issue that needs to be considered initially as well. Let's say they are doing well and their intestinal metaplasia is gone. Is follow-up conducted every 3 months, every 6 months, every year, or what?

Dr DeMeester. Once all the intestinal metaplasia has been eradicated, then follow-up is conducted at 3 months, 6 months, and then annually.

Dr Donald Low (Seattle, Wash). This is an important topic and an evolving issue in surgery. I have 2 quick questions.

Endoscopic techniques are playing a larger and larger role in virtually everything we do in the upper gastrointestinal tract. Who is doing your interventional endoscopic procedures at the University of Southern Califormia? Is it the surgeons or is it the gastroenterologist?

The second question concerns assessment and decision-making. We agree with you that treatment has to be individualized. Are all your patients, especially those with intramucosal cancer, being reviewed at your tumor boards?

Dr DeMeester. All these patients are treated by us as the surgeons, and so we do all our own endoscopic procedures, our own endoscopy follow-up, and so forth. There is no tumor board review. We make the decision with the patient on which way to go. 